Internat. J. Math. \& Math. Sci.

Vol. 22, No. 4 (1999) 705-707

S 0161-1712<99 $22705-6$

(C) Electronic Publishing House

\title{
REGULARITY OF CONSERVATIVE INDUCTIVE LIMITS
}

\author{
JAN KUCERA
}

(Received 31 July 1998)

\begin{abstract}
A sequentially complete inductive limit of Fréchet spaces is regular, see [3]. With a minor modification, this property can be extended to inductive limits of arbitrary locally convex spaces under an additional assumption of conservativeness.
\end{abstract}

Keywords and phrases. Regular and conservative inductive limits of locally convex spaces.

1991 Mathematics Subject Classification. Primary 46A13; Secondary 46A30.

Throughout the paper $E_{1} \subset E_{2} \subset \cdots$ is a sequence of Hausdorff locally convex spaces with continuous identity maps id : $E_{n} \rightarrow E_{n+1}, n \in N$. Their respective topologies are denoted by $\tau_{n}$. The topology of their inductive limit ind $E_{n}$ is denoted by $\tau=\operatorname{ind} \tau_{n}$.

We will use a result from [1, Cor. IV. 6.5]. It reads:

If $F$ as well as all spaces $E_{n}$ are Fréchet and $T: F \rightarrow$ ind $E_{n}$ is a linear map with a closed graph, then there is $n \in N$ such that $T$ is a continuous map of $F$ into $E_{n}$.

According to [2, Sec. 5.2], the space ind $E_{n}$ is called $\alpha$-regular, resp. regular, if every set bounded in ind $E_{n}$ is contained, resp. bounded, in some constituent space $E_{n}$. We will need a slightly modified notion of regularity.

DEFinItIon 1. An inductive limit ind $E_{n}$ is quasi $\alpha$-regular, resp. quasi regular, if every set bounded in ind $E_{n}$ is a subset of a $\tau$-closure of a set contained, resp. bounded, in some constituent space $E_{n}$.

DEFINITION 2. An inductive limit ind $E_{n}$ is called conservative if for every linear subspace $F \subset$ ind $E_{n}$, we have

$$
\text { ind }\left(F \cap E_{n}, \tau_{n}\right)=\left(F \text {, ind } \tau_{n}\right) .
$$

LEMmA. Let a locally convex (Hausdorff) space E be sequentially complete, and B be a balanced, bounded, closed, and convex set in $E$. Then the linear span $F$ of B, equipped with the topology generated by the Minkowski functional of $B$, is a Banach space and the identity map id: $F \rightarrow E$ is continuous.

Proof. Clearly $F$ is a normed space and id $F \rightarrow E$ is continuous.

To prove the completeness of $F$, take a Cauchy sequence $\left\{x_{n}\right\}$ in $F$. Since id : $F \rightarrow E$ is continuous, $\left\{x_{n}\right\}$ is Cauchy in $E$. Hence it converges to some $x \in E$. The set $\bigcup\left\{x_{n} ; n \in\right.$ $N$, which is bounded in $F$, is contained in some $\alpha B$. Since the set $\alpha B$ is closed in $E$, we have $x \in \alpha B \subset F$.

For any 0 -nbhd $\lambda B, \lambda>0$, in $F$, there exists $k \in N$ such that $m, n>k$ imply $x_{n}-x_{m} \in$ $\lambda B$. If we let $m \rightarrow \infty$, we get $x_{n}-x \in \lambda B$ for $n>k$, i.e., $x_{n} \rightarrow x$ in $F$. 
Proposition 1. Any sequentially complete ind $E_{n}$ is quasi $\alpha$-regular.

Proof. Let a set $A$ be bounded in ind $E_{n}$. Denote by $B$ its balanced, convex, $\tau$ closed hull, and by $F$ the linear span of $B$ with the same topology $\gamma$ as in the Lemma. We know that $F$ is a Banach space.

For any $n \in N$, denote by $G_{n}$ the completion of the normed space $\left(F \cap E_{n}, \gamma\right)$. Then $G_{n} \subset F$ and $F$ equals strict inductive limit ind $G_{n}$. Since $B$ is bounded in $F$, it is bounded in ind $G_{n}$. Hence, by [1, Cor. IV. 6.5], $B$ is bounded in some $G_{n}$.

Finally, $A \subset B$ and $B$ is a $\gamma$-closure of a set $V=\bigcup\left\{E_{n} \cap \lambda B ; 0<\lambda<1\right\}$ in $F \cap E_{n}$. Hence $A$ is also a subset of the $\tau$-closure of $V$ in ind $E_{n}$.

Proposition 2. Let ind $E_{n}$ be sequentially complete and conservative. Then every set $A \subset E_{1}$, which is bounded in ind $E_{n}$ is also bounded in some constituent space $E_{n}$.

Proof. Take such $A$ and assume that it is not bounded in any $E_{n}$. Then for any $n \in N$, there exists a balanced convex 0-nbhd $U_{n}$ in $E_{n}$ which does not absorb $A$. For any $m, n \in N$, choose $a_{m, n} \in A$ such that $a_{m, n} \notin m U_{n}$. Denote by $B$ the $\tau$-closure of the convex balanced hull of $\bigcup\left\{a_{m, n} ; m, n \in N\right\}$ and by $F$ the linear span of $B$. For any $m, n \in N$, there exists $f_{m, n} \in$ (ind $\left.E_{n}\right)^{\prime}$, (the dual of ind $E_{n}$ ), such that $f_{m, n}\left(a_{m, n}\right) \neq 0$. Put $V_{m, n}=\left\{x \in F ;\left|f_{m, n}(x)\right| \leq 1\right\}$ and denote by $F_{n}$ the linear space $F$ equipped with the topology generated by $\left\{U_{m} ; m \geq n\right\} \bigcup\left\{V_{m, n} ; m, n \in N\right\}$. Then each $F_{n}$ is a metrizable Hausdorff locally convex space and its completion $G_{n}$ is a Fréchet space.

Finally, let $H$ be the space $F$ equipped with the topology generated by the Minkowski functional of $B$. The set $B$ is bounded in ind $E_{n}$, hence, by the Lemma, $H$ is Banach space and the identity map id : $H \rightarrow$ ind $E_{n}$ is continuous.

Since ind $E_{n}$ is conservative and $F \subset$ ind $E_{n}$, we have

$$
\text { ind }\left(F, \tau_{n}\right)=\left(F \text {,ind } \tau_{n}\right) \text {. }
$$

For any $n \in N$, the identity maps $\left(F, \tau_{n}\right) \rightarrow F_{n} \rightarrow G_{n}$ are continuous. Hence

$$
\text { id }: \text { ind }\left(F, \tau_{n}\right) \longrightarrow \text { ind } G_{n}
$$

is continuous, too. Then, the continuity of id :H $\rightarrow$ ind $E_{n}$ implies the continuity of id : $H \rightarrow\left(F\right.$,ind $\left.\tau_{n}\right)$. By (2) and (3), we finally get the continuity of id: $H \rightarrow$ ind $G_{n}$.

By [1, Cor. IV. 6.5], there exists $n \in N$ such that id: $H \rightarrow G_{n}$ is continuous. Since the set $B$ is bounded in $H$ and contained in $F_{n}$, it is bounded in $G_{n}$, and also bounded in $F_{n}$. But then $B$, as well as its subset $A$, are absorbed by the 0 -nbhd $V_{n}$ in $F_{n}$, a contradiction.

By combining Propositions 1 and 2, we get

THEOREM. Any sequentially complete conservative ind $E_{n}$ is quasi regular.

COROLlARY. If moreover each space $E_{n}$ in the above Theorem is closed in ind $E_{n}$, then ind $E_{n}$ is regular.

\section{REFERENCES}

[1] M. De Wilde, Closed graph theorems and webbed spaces, Research Notes in Mathematics, vol. 19, Pitman (Advanced Publishing Program), London, Boston, MA, 1978. MR 81j:46013. Zbl 373.46007. 
[2] K. Floret, Lokalkonvexe Sequenzen mit kompakten Abbildungen, J. Reine Angew. Math. 247 (1971), 155-195. MR 44\#4478. Zbl 209.43001.

[3] J. Kucera, Sequential completeness of LF-spaces, to appear in Czechoslovak Math. J.

Kucera: DePartment of Mathematics, WASHington STATE University, PUllman, WASHINGTON 99164-3113, USA 


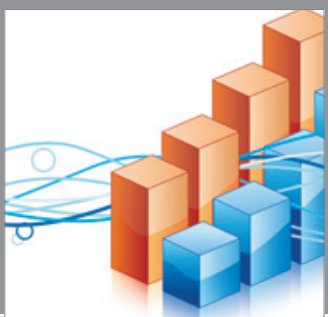

Advances in

Operations Research

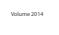

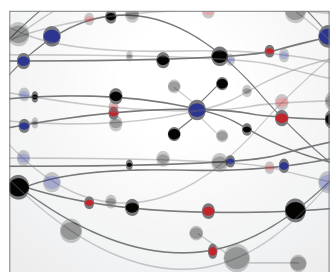

\section{The Scientific} World Journal
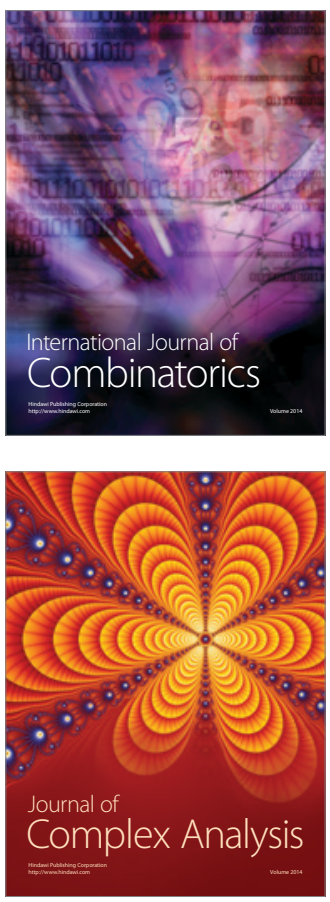

International Journal of

Mathematics and

Mathematical

Sciences
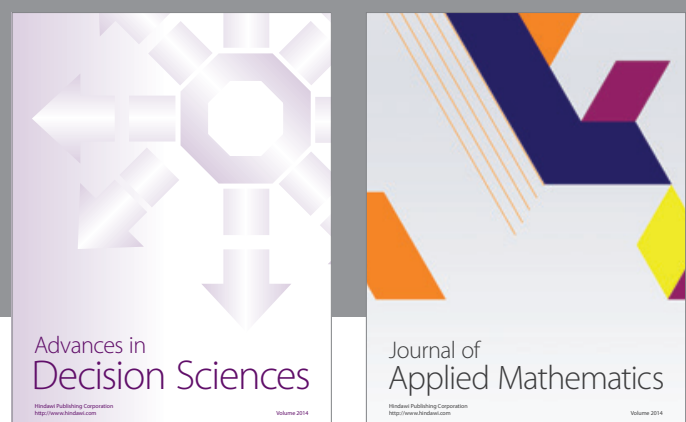

Journal of

Applied Mathematics
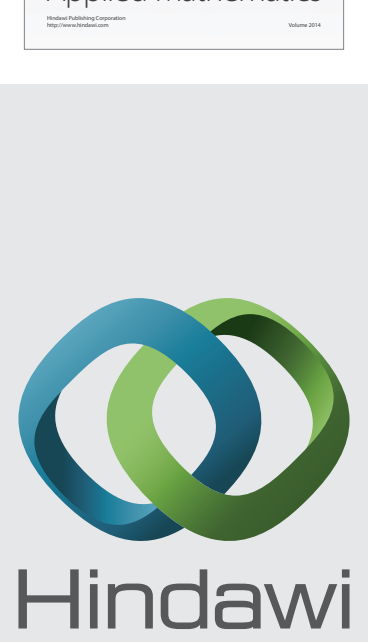

Submit your manuscripts at http://www.hindawi.com
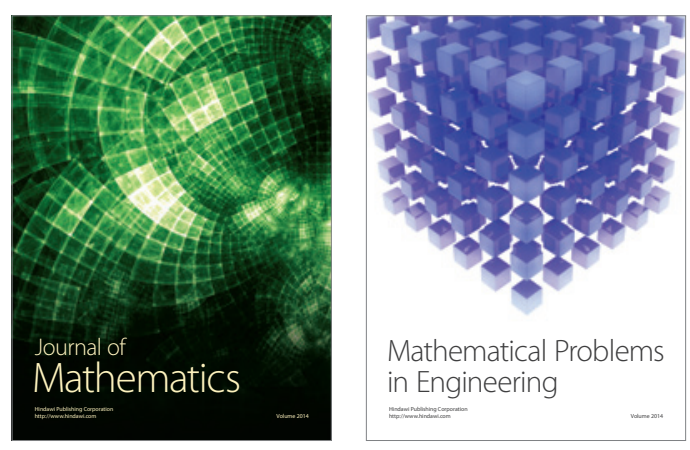

Mathematical Problems in Engineering
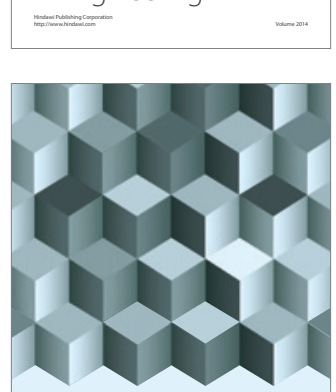

Journal of

Function Spaces
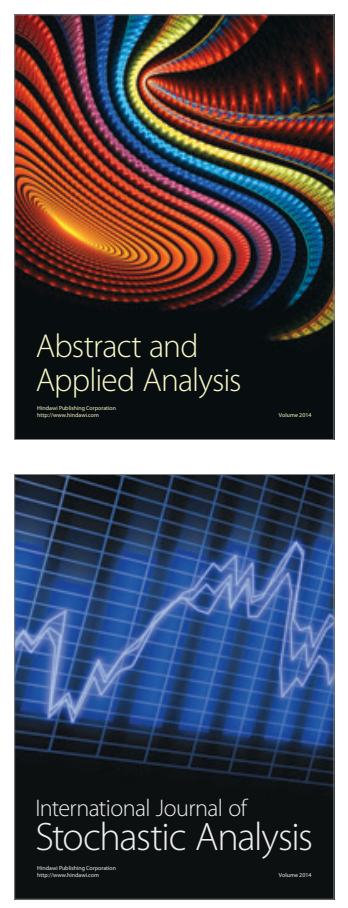

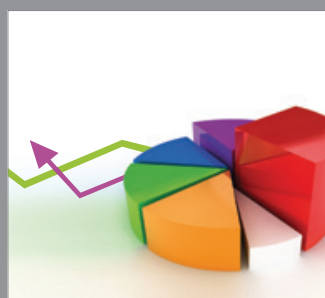

ournal of

Probability and Statistics

Promensencen
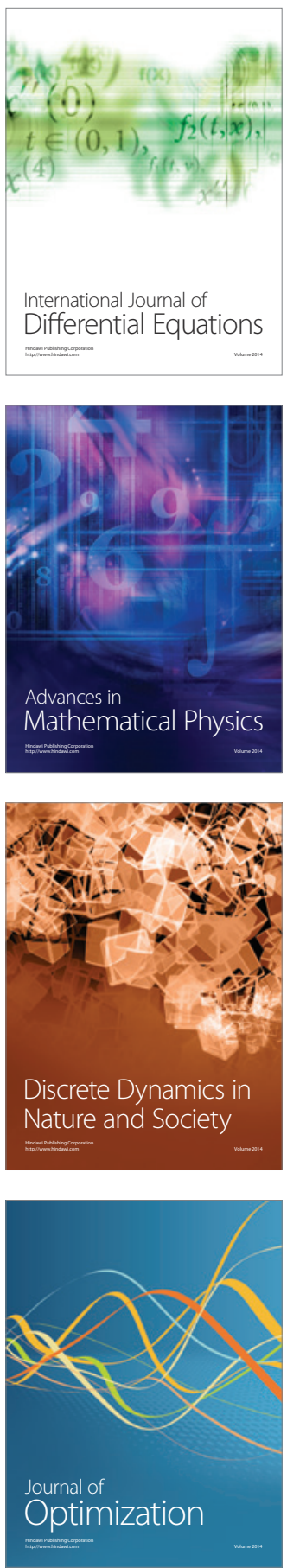